\title{
Outcomes and Nephrotoxicity Associated with Vancomycin Treatment in Patients 80 Years and Older
}

\author{
Yunchao Wang (D) \\ Ning Dai ${ }^{1}$ \\ Wei $\mathrm{Wei}^{2}$ \\ Chunyan Jiang'
}

'Department of Internal Medicine and Geriatrics, Beijing Friendship Hospital, Capital Medical University, Beijing, People's Republic of China; ${ }^{2}$ Department of Clinical Epidemiology and EBM, Beijing Friendship Hospital, Capital Medical University, Beijing, People's Republic of China
Correspondence: Chunyan Jiang Department of Internal Medicine and Geriatrics, Beijing Friendship Hospital, Capital Medical University, No. 95,

Yong'an Road, Xicheng District, Beijing, 100050, People's Republic of China

$\mathrm{Tel}+861063137648$

$\mathrm{Fax}+861063138795$

Email chunyan_jiang@ccmu.edu.cn
Purpose: This retrospective observational study investigated the efficacy and safety of vancomycin to treat patients aged 80 years and older. In particular, the associations between vancomycin trough concentration (VTC) and treatment outcomes or nephrotoxicity were explored.

Patients and Methods: Patients aged $\geq 80$ years had received $\geq 3$ vancomycin treatments and $\geq 1$ detection of VTC. Treatment outcomes were defined as success or failure. Nephrotoxicity was considered an increase in serum creatinine $\geq 44.2 \mathrm{mmol} / \mathrm{L}$, or $50 \%$ above baseline, for $\geq 2$ consecutive days. Univariate and multivariate analyses were performed to identify risk factors for treatment failure and nephrotoxicity.

Results: Of 349 patients, 120 (34.4\%) experienced treatment failure. For patients with VTCs at $<10,10-15,15-20$, and $\geq 20 \mu \mathrm{g} / \mathrm{mL}$, the clinical response rates were, respectively, 77.8 , $77.0,80.5$, and $61.0 \%$; the 30 -day mortality rates were $2.8,15.0,15.3$, and $37.8 \%$; and the rates of persistent bacteremia were $16.7,12.4,11.9$, and $11.0 \%$. The multivariate analysis indicated that blood urea nitrogen $\geq 11 \mathrm{~g} / \mathrm{dL}$ and heart failure were independently associated with treatment failure; but not VTC ( $P=0.004,0.016,0.828$, respectively). During vancomycin treatment, $42(12.0 \%)$ patients experienced nephrotoxicity with recovery time $7.5 \pm$ 4.5 days. Fewer than half of patients with nephrotoxicity recovered after suspending vancomycin application. The variables found independently associated with increased nephrotoxicity were: VTC $\geq 15 \mu \mathrm{g} / \mathrm{mL}$; treatment duration $\geq 15 \mathrm{~d}$; and concomitant aminoglycosides administration $(P=0.024,0.035,0.029)$.

Conclusion: In patients aged 80 years and older, elevated VTC level was not associated with favorable treatment outcomes. Patients with VTC $\geq 20 \mu \mathrm{g} / \mathrm{mL}$ appear to suggest a worsened prognosis compared with lower VTCs. The risk of nephrotoxicity increases with elevated VTC, longer treatment time, and concomitant aminoglycoside administration. Keywords: elderly, vancomycin trough concentration, outcome, nephrotoxicity

\section{Plain Language Summary}

Vancomycin is commonly used to treat Gram-positive bacterial infections that resist antibiotics, but can be detrimental to kidney function. We hypothesized that it may be more difficult for very old patients to eliminate vancomycin quickly, which puts their renal system at risk. This study reviewed evidence to analyze the safety and efficacy of vancomycin, in 349 patients aged 80 years or older who had received at least 3 vancomycin treatments to treat recalcitrant bacterial infections of various types. Nephrotoxicity (a poisonous effect on the kidneys) was also measured, based on a certain level of blood serum creatinine lasting at least 2 days. The results showed that bacterial infection was not controlled in about $34 \%$ of 
patients, but this lack of improvement was due to factors other than vancomycin (such as heart failure). About $12 \%$ experienced nephrotoxicity which was related to the vancomycin treatment, among whom fewer than half recovered, especially those with higher VTC, duration of treatment, and simultaneous use of another antibiotic (aminoglycosides). A favorable clinical response to vancomycin was less likely as VTC increased, and indeed VTC $\geq 20 \mu \mathrm{g} / \mathrm{mL}$ indicated a worse prognosis. Therefore, vancomycin should be applied with caution in the elderly. Treatment decisions should consider multiple factors, including a suitably low VTC, to improve vancomycin efficacy and reduce the risk of nephrotoxicity.

\section{Introduction}

Vancomycin is a glycopeptide antibiotic with a history that can be traced back to the 1950s when it was discovered produced by Streptomyces orientalis in soil. ${ }^{1}$ It is one of the most commonly prescribed drugs, and for decades has been the main treatment for patients with suspected or documented antibiotic-resistant Gram-positive infections. $^{2-4}$ The recommended pharmacokineticpharmacodynamic target $\mathrm{AUC} / \mathrm{MIC}$ ratio for vancomycin is $>400$ (ie, the ratio of the area under the serum concentration time curve [AUC] to the minimum inhibitory concentration [MIC]). This is particularly true for treating methicillin-resistant Staphylococcus aureus (MRSA) infections. ${ }^{5}$ To facilitate management and simplify vancomycin dose adjustments and monitoring, multiple organizations in 2009 recommended trough monitoring and maintaining trough concentrations between 15 and $20 \mu \mathrm{g} /$ $\mathrm{mL}$. Since these guidelines were published, several studies have evaluated the efficacy and safety of recommended vancomycin trough concentration (VTC), with conflicting results. More recent research suggested that high VTC did not correlate with any notable improvement in treatment outcomes, in either adults or children. ${ }^{6-8}$

Nephrotoxicity remains the most severe vancomycinassociated adverse effect, as reported by many studies, and is associated with increased mortality, hospital stay, and medical expense. ${ }^{9,10}$ Although vancomycin is often associated with nephrotoxicity, the direct mechanisms are controversial. Multiple studies have focused on oxidative stress as a potential mechanism of nephrotoxicity, especially involving the proximal tubule. ${ }^{11,12}$ Other studies showed that vancomycin can change the energydependent renal reabsorption function of the proximal tubule cells and alter mitochondrial function, which is also associated with vancomycin-induced renal toxicity. ${ }^{13}$
In a meta-analysis, van $\mathrm{Hal}$ et $\mathrm{al}^{10}$ reported a $5 \%$ to $43 \%$ rate of vancomycin-associated nephrotoxicity. Recently, Sinha Ray and colleagues ${ }^{14}$ conducted a meta-analysis comprising 13 studies and showed that the relative risk of nephrotoxicity during vancomycin treatment was 2.45 (OR 2.45; 95\% CI 1.69-3.55), and the attributable risk of nephrotoxicity was $59 \%$. Additionally, these studies also showing that higher VTC and longer duration of vancomycin treatment were independent risk factors associated with nephrotoxicity. ${ }^{10,14}$

Vancomycin is eliminated from the body mainly via the kidneys. The clearance is linearly related to the glomerular filtration rate, and thus reduced renal clearance may lead to increased VTC. ${ }^{15}$ Previous studies have showed that longer half-life ( $\mathrm{t} 1 / 2)$, higher volume of distribution and reduced clearance of vancomycin in older patients, compared with the younger, and this may lead to increased VTC level and the risk of nephrotoxicity. ${ }^{16-18}$ It is estimated that by 2050 the percentage of the population older than 65 years will be as high as $40 \%$, and the very old as much as $15 \% .{ }^{19}$ This demographic change will increase the number of geriatric patients in all areas of medical care. Guidelines regarding renal safety and trough concentration, and whether VTC correlates with risk of nephrotoxicity in elderly patients, are an important concern in clinical practice. This is particularly true for patients aged 80 years and older. However, studies of vancomycin treatment specifically in this group of patients are rare.

The current study investigated the efficacy and safety of vancomycin to treat patients aged 80 years and older. In particular, the associations between VTC and treatment outcomes or nephrotoxicity were explored.

\section{Patients and Methods Data Collection}

This is a retrospective observational study which includes data of patients treated at Beijing Friendship Hospital, Capital Medical University, from January 2017 to December 2019. The study was performed in accordance with the principles of the Declaration of Helsinki and was approved by the Ethics Committee of Beijing Friendship Hospital, Capital Medical University (No. 2020-P2-109$01)$. The committee waived the need for informed consent in this retrospective study with no potential harm to subjects, and permission was granted to use data for analysis and all the data is strictly confidential. 
Inpatients who met all the following criteria were included: aged $\geq 80$ years; with confirmed or suspected complicated Gram-positive infection; and with at least one detection of serum steady state VTC. Patients who received any of the following were excluded: vancomycin oral or continuous intravenous treatment; $<3$ vancomycin doses, with undetectable serum VTC; or renal replacement therapy such as hemodialysis, peritoneal dialysis, or hemofiltration before or during vancomycin treatment. Also excluded were patients with severe chronic renal dysfunction (creatinine clearance ratio $<20 \mathrm{~mL} / \mathrm{min}$ ), or in whom renal injury occurred prior to vancomycin administration (see below); or lacking information to evaluate the treatment effect and nephrotoxicity. Acute kidney injury was defined as an increase in serum creatinine ( $\mathrm{SCr}$ ) level by $>0.3 \mathrm{mg} / \mathrm{dL}$ within 48 hours, or an increase in $\mathrm{SCr}$ level $>1.5$-fold the baseline, which was known or presumed to have occurred within the preceding 7 days, or urine output $<0.5 \mathrm{~mL} / \mathrm{kg} / \mathrm{h}$ for 6 hours. ${ }^{9}$

The following data were collected from the patients' medical records: demographics (age, gender), body weight and body mass index (BMI), and comorbid disease. Noted infections were: pneumonia, blood stream infection, endocarditis, osteomyelitis, meningitis, urinary infection, biliary system infection, celiac infection, and skin or soft tissue infection. Laboratory data included $\mathrm{SCr}$, blood urea nitrogen (BUN), serum albumin, hemoglobin, and estimated glomerular filtration rate (eGFR). The particulars of vancomycin therapy were dosage, treatment duration, and VTC. Noted etiologies were MRSA, coagulasenegative Staphylococcus, Streptococcus, Enterococcus, and minimum inhibitory concentrations of these bacteria. Drugs that influence renal function were recorded, including aminoglycosides, diuretics, angiotensin converting enzyme inhibitors/angiotensin receptor blockers (ACEI/ ARB), cyclooxygenase 2 inhibitors, and nonsteroidal antiinflammatory drugs (NSAIDs).

Finally, data regarding nephrotoxicity and treatment outcome were gathered. VTCs were obtained within 72 hours of treatment (before the fourth and fifth vancomycin administration). During vancomycin therapy, the SCr, and VTC were monitored every 3 to 4 days, and endogenous creatinine clearance was tested weekly.

\section{Treatment Outcome Evaluation}

Treatment outcomes were classified as either success or failure, based on a composite evaluation of clinical response, persistent bacteremia, and 30-day mortality.
Clinical response was defined as with response or no response, based on infection signs and symptoms, laboratory tests, and medical images. Response was defined as infection signs and symptoms, laboratory tests, and medical images relief or cure after treatment. No response was defined as no improvement in infection signs or symptoms, or worsening or even appearance of new symptoms associated with the original infection. Persistent bacteremia meant lasting at least 7 days after the initial treatment of vancomycin. The 30-day mortality was the mortality rate at 30 days after vancomycin therapy. Treatment success was considered treatment response, microbiological eradication, and survival within 30 days after the initial vancomycin treatment. Conversely, treatment failure was defined as no clinical response, or persistent bacteremia, or death within 30 days after initial vancomycin treatment. $^{6,8,20}$

\section{Evaluation of Nephrotoxicity}

Vancomycin-associated nephrotoxicity was defined as either an increase in $\mathrm{SCr} \geq 44.2 \mathrm{mmol} / \mathrm{L}(0.5 \mathrm{mg} / \mathrm{dL})$, or $50 \%$ above baseline, from initiation of vancomycin to 72 hours after completion of therapy, excluding other causes of acute kidney injury. ${ }^{8}$

\section{Statistical Analysis}

Patients were classified as vancomycin treatment success or failure, or with or without vancomycin-associated nephrotoxicity, and their demographic and clinical variables compared. Categorical data were analyzed using the chi-squared test or Fisher's exact test, and continuous data were examined using Student's $t$-test or a MannWhitney $U$-test for small sample parametric or nonparametric variables. Cox multiple variable regression analyses were performed to identify the risk factors for vancomycin outcome and nephrotoxicity. All variables with a $P$-value $<0.05$ in the univariate analysis were included in the final regression model. A $P$-value $<0.05$ was considered statistically significant. Statistical analyses were performed using SPSS software, version 25.0 (SPSS, Chicago, IL, USA).

\section{Results}

\section{Demographics and Clinical} Characteristics

The study population comprised 349 elderly ( $\geq 80$ years) patients with a median age of $88.0(84.0,91.0)$ years; 
$71.3 \%$ were men (Table 1$)$. More than half $(55.7 \%)$ had a BMI of $18.5-24.9 \mathrm{~kg} / \mathrm{m}^{2}$. Most of the patients had at least one comorbidity, including hypertension (71.1\%), chronic heart failure $(54.4 \%)$, diabetes $(37.0 \%)$, chronic kidney dysfunction (24.1\%), malignancies (17.5\%), and respiratory diseases (15.2\%).

Most of the subjects (97.4\%) had only one infection site, while the rest had 2 or more (Table 1). The main infection sites were the respiratory system $(80.8 \%)$, urinary $(8.0 \%)$, blood stream $(6.3 \%)$, biliary system $(4.0 \%)$, celiac $(2.0 \%)$, and skin/soft tissue (1.7\%). Gram-positive bacteria were detected in the blood or body fluid cultures of $35.5 \%$, including $47,43,29$, and 2 strains of Enterococcus, MRSA, coagulase-negative Staphylococcus, and Streptococcus, respectively.

\section{Treatment Outcomes}

Among the 349 patients, 229 (65.6\%) and 120 (34.4\%) experienced treatment success and failure, respectively (Table 1). There were significant differences between the success and failure treatment groups only with regard to the following: rate of chronic heart failure $(P=0.029)$, VTC level $(P=0.012)$, vancomycin admission daily dose $(P=0.045)$, estimated glomerular filtration rate (eGFR; $P=0.049)$ and BUN $(P=0.041)$. Thus, these variables were entered into the multivariable logistic regression model (Table 2). Results of the multivariate analysis indicated that average trough concentration (OR $0.948 ; 95 \%$ CI $0.584-1.538 ; P=0.828$ ) was not significantly associated with treatment failure. Only BUN $\geq 11$ g/dL (OR 1.060; 95\% CI 1.019-1.103; $P=0.004$ ) and heart failure (OR 1.807; 95\% CI 1.118-2.922; $P=0.016$ ) were independently associated with treatment failure.

To observe whether targeting the recommended higher VTC $(15-20 \mu \mathrm{g} / \mathrm{mL})^{5}$ leads to improved vancomycin treatment outcomes, the patients were stratified into 4 groups based on the following VTCs: $<10,10-15,15-20$, and $\geq 20$ $\mu \mathrm{g} / \mathrm{mL}$. The clinical response rates of these groups were 77.8 , $77.0,80.5$, and $61.0 \%$ respectively; the 30 -day mortality rates were $2.8,15.0,15.3$, and $37.8 \%$; and the rates of persistent bacteremia were 16.7, 12.4, 11.9, and 11.0\% (Figure 1). The treatment outcomes of the groups with VTC $<10,10-15$, and $15-20 \mu \mathrm{g} / \mathrm{mL}$ were not significant different $(P=0.530)$ but it was worse in $\geq 20 \mu \mathrm{g} / \mathrm{mL}$ group $(P<0.000)$.

\section{Nephrotoxicity}

$\mathrm{SCr}$ and eGFR were routinely monitored in all 349 patients during the vancomycin treatment period
(Table 3). Of these, $42(12.0 \%)$ patients experienced nephrotoxicity. Among those with nephrotoxicity, 37 (88.1\%) showed a 50\% increase in baseline SCr, and 27 (64.3\%) with a $\mathrm{SCr}$ increase of $\geq 0.5 \mathrm{mg} / \mathrm{dL}$. (There were 22 patients with not only a $50 \%$ increase in baseline $\mathrm{SCr}$, but also an $\mathrm{SCr}$ increase of $\geq 0.5 \mathrm{mg} / \mathrm{dL}$ ). The time to nephrotoxicity was $7.5 \pm 4.5$ days; in $59.5 \%$, nephrotoxicity occurred during the first week of treatment. After suspending vancomycin application, 20 (47.6\%) patients with renal dysfunction returned to baseline level.

A positive association was observed between VTC and nephrotoxicity (Figure 2). Nephrotoxicity increased with VTC. The rates of nephrotoxicity were 9.5, 21.4, 28.6, and $40.5 \%$ at VTCs at $<10,10-15,15-20$, and $\geq 20 \mu \mathrm{g} / \mathrm{mL}$, respectively $(P=0.045)$. The univariate analysis suggested that the following were associated with vancomycinrelated nephrotoxicity: VTC, treatment duration, hemoglobin level, eGFR, and application of aminoglycosides (Table 3).

These variables were introduced into the multivariate logistic regression model (Table 4), and the results indicated that $\mathrm{VTC} \geq 15 \mu \mathrm{g} / \mathrm{mL}$ (OR $2.469 ; 95 \%$ CI 1.127-5.408; $P=0.024$ ), treatment duration $\geq 15 \mathrm{~d}$ (OR 3.241; 95\% CI 1.086-9.672; $P=$ 0.035), and concomitant aminoglycosides (OR 5.147; 95\% CI 1.187-22.314; $P=0.029$ ) were independently associated with increased nephrotoxicity during treatment with vancomycin.

\section{Discussion}

The present study evaluated the treatment outcomes and nephrotoxicity of patients aged 80 or more years, with common characteristics such as multiple comorbidities and varying degrees of renal dysfunction. Among the 349 patients enrolled, vancomycin therapy failed for 120 (34.4\%), and 42 (12.0\%) suffered nephrotoxicity associated with vancomycin. Higher levels of VTC were not associated with better treatment outcomes and did increase the risk of vancomycin-induced nephrotoxicity. In contrast, patients with too high VTC ( $\geq 20 \mu \mathrm{g} / \mathrm{mL})$ seems have lower clinical response, and highest 30-day mortality rate.

The vancomycin therapeutic guidelines published in 2009 recommended targeting VTC to above $10 \mu \mathrm{g} / \mathrm{mL}$ to prevent drug-resistance and maintaining VTC at 15 to 20 $\mu \mathrm{g} / \mathrm{mL}$ for complicated infections to improve vancomycin efficacy. ${ }^{5}$ However, the present study found that higher VTC levels did not correlate with any notable improvement in treatment outcomes. Rather, a VTC higher than 20 $\mu \mathrm{g} / \mathrm{mL}$ was associated with worse prognosis. 
Table I Demographics and Clinical Characteristics of the Study Population ${ }^{\text {a }}$

\begin{tabular}{|c|c|c|c|c|}
\hline & \multirow[b]{2}{*}{ Total } & \multicolumn{3}{|c|}{ Vancomycin Treatment } \\
\hline & & Success & Failure & $\mathbf{P}$ \\
\hline Subjects, $n$ & 349 & 229 & 120 & \\
\hline Age, y, M (QI, Q3) & $88.0(84.0,91.0)$ & $88.0(84.0,91.0)$ & $88.0(85.0,91.0)$ & 0.732 \\
\hline Male, n (\%) & $249(71.3)$ & $168(73.4)$ & $81(67.5)$ & 0.250 \\
\hline Weight, kg ${ }^{\mathrm{b}}$ & $61.0(50.0,70.0)$ & $60.0(50.0,70.0)$ & $62.4(54.3,70.0)$ & 0.771 \\
\hline Weight, kg, n (\%) & & & & 0.180 \\
\hline$<55$ & $103(35.9)$ & $73(38.2)$ & $30(31.3)$ & 0.245 \\
\hline $55-65$ & $82(28.6)$ & $48(25.1)$ & $34(35.4)$ & 0.069 \\
\hline$\geq 65$ & $102(35.5)$ & $70(36.6)$ & $32(33.3)$ & 0.580 \\
\hline BMI, kg/m ${ }^{2}$ & $22.4 \pm 3.9$ & $22.3 \pm 4.1$ & $22.5 \pm 3.7$ & 0.656 \\
\hline BMI, kg/m², n (\%) & & & & 0.414 \\
\hline$<18.5$ & $49(17.1)$ & $36(18.8)$ & $13(13.5)$ & 0.260 \\
\hline $18.5-24.9$ & $160(55.7)$ & $101(52.9)$ & $59(61.5)$ & 0.732 \\
\hline $25.0-29.9$ & $72(25.1)$ & $49(25.7)$ & $23(24.0)$ & 0.754 \\
\hline$\geq 30.0$ & $6(2.1)$ & $5(2.6)$ & I (I.0) & 0.658 \\
\hline Comorbid conditions ${ }^{\mathrm{c}}$, n (\%) & & & & 0.284 \\
\hline Chronic pulmonary disease & $53(15.2)$ & $35(15.3)$ & $18(15.0)$ & 0.944 \\
\hline Hypertension & $248(7 I .1)$ & 167 (72.9) & $81(67.5)$ & 0.288 \\
\hline Heart failure & $190(54.4)$ & II 5 (50.2) & $75(62.5)$ & 0.029 \\
\hline Type 2 diabetes & $129(37.0)$ & $84(36.7)$ & $45(37.5)$ & 0.880 \\
\hline CKD & $84(24.1)$ & $46(20.1)$ & $38(31.7)$ & 0.016 \\
\hline Malignancies & $61(17.5)$ & $35(15.3)$ & $26(21.7)$ & 0.136 \\
\hline Infection site ${ }^{d}, \mathrm{n}(\%)$ & & & & 0.169 \\
\hline Nosocomial pneumonia & $282(80.8)$ & $186(8 \mid .2)$ & $96(80.0)$ & 0.783 \\
\hline Blood stream infection & $22(6.3)$ & II (4.8) & II (9.2) & 0.111 \\
\hline Endocarditis & Nil & Nil & Nil & - \\
\hline Osteomyelitis & Nil & Nil & Nil & - \\
\hline Meningitis & Nil & Nil & Nil & - \\
\hline Urinary & $28(8.0)$ & $14(6.1)$ & 14 (II.7) & 0.070 \\
\hline Biliary system & $14(4.0)$ & $12(5.2)$ & $2(1.7)$ & 0.106 \\
\hline Celiac & $7(2.0)$ & $5(2.2)$ & $2(1.7)$ & 0.744 \\
\hline Skin/soft tissue & $6(1.7)$ & $4(1.7)$ & $2(1.7)$ & 0.956 \\
\hline Bacterial strains, n (\%) & & & & 0.620 \\
\hline MRSA & $43(12.3)$ & $20(8.7)$ & $23(19.2)$ & 0.055 \\
\hline CNS & $29(8.3)$ & $18(7.9)$ & II (9.2) & 0.675 \\
\hline Streptococcus & $2(0.6)$ & $\mathrm{I}(0.4)$ & $\mathrm{I}(0.8)$ & 0.650 \\
\hline Enterococcus & $47(13.5)$ & $26(11.4)$ & $21(17.5)$ & 0.110 \\
\hline VTC, $\mu g / m L, M(\min , \max )$ & $17.0(4.2,62.5)$ & $16.5(4.2,53.6)$ & I8.I $(6.7,62.5)$ & 0.035 \\
\hline VTC, $\mu g / m L, n(\%)$ & & & & 0.012 \\
\hline$<10$ & $36(10.3)$ & $23(10.0)$ & $13(10.8)$ & 0.818 \\
\hline $10-15$ & $113(32.4)$ & $77(33.6)$ & $36(30.0)$ & 0.492 \\
\hline $15-20$ & 118 (33.8) & $86(37.6)$ & $32(26.7)$ & 0.041 \\
\hline$\geq 20$ & $82(23.5)$ & $43(18.8)$ & $39(32.5)$ & 0.004 \\
\hline
\end{tabular}

(Continued) 
Table I (Continued).

\begin{tabular}{|c|c|c|c|c|}
\hline & \multirow[b]{2}{*}{ Total } & \multicolumn{3}{|c|}{ Vancomycin Treatment } \\
\hline & & Success & Failure & $\boldsymbol{P}$ \\
\hline Vancomycin dose, g/d, n (\%) & & & & 0.045 \\
\hline$<1.5$ & $247(70.8)$ & I54 (67.2) & $93(77.5)$ & \\
\hline$\geq 1.5$ & $102(29.2)$ & $75(32.8)$ & $27(22.5)$ & \\
\hline SCr, g/dL, M (QI, Q3) & $67.0(54.0,91.4)$ & $65.7(54.0,85.7)$ & $72.7(53.1,97.1)$ & 0.219 \\
\hline eGFR, mL/min/l.73 $\mathrm{m}^{2}, \mathrm{n}(\%)$ & & & & 0.049 \\
\hline$\geq 90$ & $81(23.2)$ & $50(2 \mid .8)$ & $31(25.8)$ & 0.401 \\
\hline $60-89$ & $201(57.6)$ & $142(62.0)$ & $59(49.2)$ & 0.021 \\
\hline $20-59$ & $67(19.2)$ & $37(16.2)$ & $30(25.0)$ & 0.046 \\
\hline BUN, g/dL, n (\%) & & & & 0.041 \\
\hline$<11$ & $272(77.9)$ & $186(8 \mid .2)$ & $86(71.7)$ & \\
\hline$\geq 11$ & $77(22.1)$ & $43(18.8)$ & $34(28.3)$ & \\
\hline Albumin, $g / L$ & $30.9 \pm 4.4$ & $31.2 \pm 4.4$ & $30.6 \pm 4.5$ & 0.223 \\
\hline Hemoglobin, g/dL, M (QI, Q3) & $101.0(90.0,119.0)$ & $102.0(91.0,121.0)$ & $100.0(86.3,115.7)$ & 0.069 \\
\hline
\end{tabular}

Notes: ${ }^{a}$ Shown as $\mathrm{n}(\%)$, unless indicated otherwise; body weight was not available for 38 patients in success group and 24 in failed group; ${ }^{\circ}$ One patient may have more than one comorbid disease; ${ }^{d}$ One patient may have more than one underlying infection site and/or more than one indication.

Abbreviations: BMI, body mass index; BUN, blood urea nitrogen; CKD, chronic kidney disease; CNS, coagulase negative staphylococcus; eGFR, estimated glomerular filtration rate; MRSA, methicillin-resistant Staphylococcus aureus; VTC, vancomycin trough concentration.

Indeed, recent studies regarding the association between VTC and treatment outcomes are inconsistent. Huang et $\mathrm{al}^{21}$ reported that in 50 patients aged at least 80 years, the clinical efficacy of vancomycin treatment was $74.0 \%$, and there was no significant difference in rates of success or failure between groups above or below $15 \mu \mathrm{g} /$ $\mathrm{mL}$ VTC. The results of Hermsen et $\mathrm{al}^{22}$ were similar for patients treated for MRSA infections. Furthermore, a meta-analysis (4 prospective and 12 retrospective cohort studies) found no significant benefit in mortality or treatment outcomes for VTCs $\geq 15 \mathrm{mg} / \mathrm{L}$ relative to lower levels, although the microbiologic control rate was higher. ${ }^{23}$ Another meta-analysis involving patients with MRSA infections found no differences in outcomes or mortality at the same VTC cutoff. $^{24}$

In contrast, Kullar et $\mathrm{al}^{25}$ found that VTC $<15 \mu \mathrm{g} / \mathrm{mL}$ was an independent predictor of treatment failure in 320 patients with MRSA infections, and Cheong et $\mathrm{al}^{26}$ showed a significant association between VTC and response to treatment, also of MRSA infections. In the latter, the VTC was higher in responders $(11.64 \pm 1.50$ $\mu \mathrm{g} / \mathrm{mL})$ than non-responders $(9.25 \pm 1.59 \mu \mathrm{g} / \mathrm{mL} ; P=$ $0.036) .{ }^{26}$ Wei et $\mathrm{al}^{6}$ reported that admission to the intensive care unit was associated with vancomycin treatment

Table 2 Multivariate Logistic Regression Analysis of Factors Associated with Vancomycin Treatment Outcomes

\begin{tabular}{|c|c|c|c|c|}
\hline & $\boldsymbol{\beta}$ & SE & OR $(95 \% \mathrm{Cl})$ & $\boldsymbol{P}$ \\
\hline VTC $(\mu g / m L)^{a}$ & -0.054 & 0.247 & $0.948(0.584-1.538)$ & 0.828 \\
\hline Daily dose, $g / d^{b}$ & 0.510 & 0.275 & $1.665(0.972-2.852)$ & 0.063 \\
\hline Heart failure ${ }^{c}$ & 0.592 & 0.245 & 1.807 (1.1 I8-2.922) & 0.016 \\
\hline BUN, g/dL ${ }^{d}$ & 0.059 & 0.020 & $1.060(1.019-1.103)$ & 0.004 \\
\hline \multicolumn{5}{|c|}{ eGFR, mL/min/l.73 m², n (\%) } \\
\hline Reference, $\geq 90$ & - & - & - & - \\
\hline $60-90$ & 0.397 & 0.384 & $1.487(0.700-3.158)$ & 0.302 \\
\hline $20-60$ & -0.340 & 0.316 & $0.712(0.383-1.323)$ & 0.283 \\
\hline
\end{tabular}

Notes: ${ }^{a} \mathrm{VTC}$ was categorized as $<15 \mu \mathrm{g} / \mathrm{mL}$ and $\geq 15 \mu \mathrm{g} / \mathrm{mL}$; ${ }^{\mathrm{b}}$ Daily dose was categorized as $<1.5 \mathrm{~g} / \mathrm{d}$ or $\geq 1.5 \mathrm{~g} / \mathrm{d}$; ${ }^{\mathrm{C}} \mathrm{Heart}$ failure was categorized as with or without; ${ }^{\mathrm{d}} \mathrm{BUN}$ was categorized as $<11 \mathrm{~g} / \mathrm{dL}$ and $\geq 11 \mathrm{~g} / \mathrm{dL}$.

Abbreviations: $\beta$, regression coefficient; $\mathrm{BUN}$, blood urea nitrogen; $\mathrm{Cl}$, confidence interval; OR, odds ratio; SE, standard error; VTC, vancomycin trough concentration. 


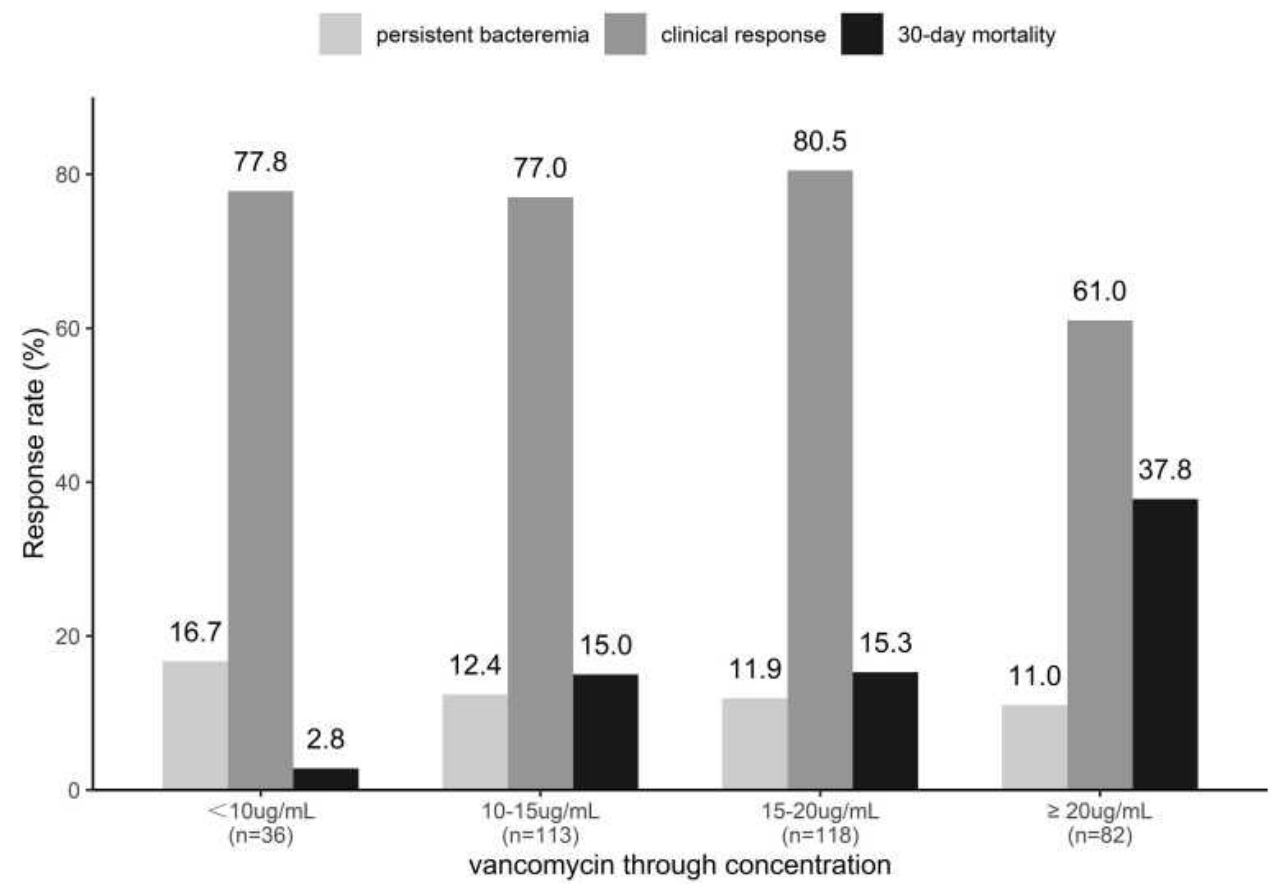

Figure I Treatment outcomes of the groups with VTCs $<10,10-15,10-20$ and $\geq 20 \mu \mathrm{g} / \mathrm{mL}$.

failure in patients with MRSA infections (OR, 3808; 95\% CI 1714-8465; $P=0.001)$. Moore et $\mathrm{al}^{27}$ showed that the APACHE II (Acute Physiology and Chronic Health Evaluation II) score was important in predicting vancomycin failure. In addition, other studies of vancomycin treatment outcomes showed that disease state and infection site, such as pneumonia and endocarditis, were predictors of vancomycin failure. ${ }^{28}$ Our study showed that concomitant with heart failure and blood urea nitrogen $\geq 11 \mathrm{~g} / \mathrm{dL}$ were independent predictors of vancomycin treatment failure in very old patients. One of the potential explanations is that the patients concomitant with chronic heart failure or elevated blood urea nitrogen $(\geq 11 \mathrm{~g} / \mathrm{dL})$ were at higher risk of exacerbation of heart failure or occurrence of acute kidney injury (AKI) or renal failure, or even occurrence of multiorgan failure, and thus were associated with vancomycin treatment failure. To the best of our knowledge, no similar findings were reported in other studies, so it needs to be further verified by prospective, large sample studies.

Although the outcomes of the guideline-recommended trough levels for suspected or documented Gram-positive infections remain controversial, there is a much stronger association between higher VTC and nephrotoxicity.,11,29 Lodise et $\mathrm{al}^{30}$ investigated VTC and nephrotoxicity based on pharmacokinetic characteristics and reported that nephrotoxicity increased significantly, by 21 to $33 \%$, for
VTCs of $10-20$ and $>20 \mu \mathrm{g} / \mathrm{mL}$, but reduced to $5 \%$ when VTC was $<10 \mu \mathrm{g} / \mathrm{mL}$. Similar results were reported by Chuma et $\mathrm{al} ;{ }^{31}$ the rate of vancomycin-associated nephrotoxicity in patients with initial trough levels $\geq 20 \mu \mathrm{g} / \mathrm{mL}$ $(31.3 \%)$ was significantly higher than that of patients with initial trough levels $<10 \mu \mathrm{g} / \mathrm{mL}(6.3 \%)$. Moreover, a study by Pan et $\mathrm{al}^{32}$ of patients aged $\geq 60$ years found that VTC $\geq 20 \mu \mathrm{g} / \mathrm{mL}$ was an independent risk factor for vancomycin-associated nephrotoxicity and led to a 3-fold increased risk of nephrotoxicity. The present study is consistent with the clinical studies described above. At each higher VTC level $(<10,10-15,15-20$, and $\geq 20 \mu \mathrm{g} / \mathrm{mL})$, the rates of nephrotoxicity also rose. VTC $\geq 15 \mu \mathrm{g} / \mathrm{mL}$ was an independent risk factor for nephrotoxicity, with a 2.46-fold increased risk of nephrotoxicity relative to the lower VTC levels. Thus, nephrotoxicity was closely related to the VTC in our elderly patients.

A meta-analysis of 15 studies compared patients with VTCs less or greater than $15 \mu \mathrm{g} / \mathrm{mL}$, and found that nephrotoxicity occurred 4 to 17 days after the start of vancomycin application. ${ }^{2,9,14,20,33}$ Another study by Hirai et $\mathrm{al}^{34}$ showed that the mean onset of vancomycinassociated nephrotoxicity was $6.9 \pm 4.9$ days, and $40.0 \%$ of patients recovered from it. Because of large interindividual variability of pharmacokinetic parameters of vancomycin in very old patients, vancomycin levels and 
Table 3 Analysis of Nephrotoxicity in Patients with Vancomycin Treatment ${ }^{\mathrm{a}}$

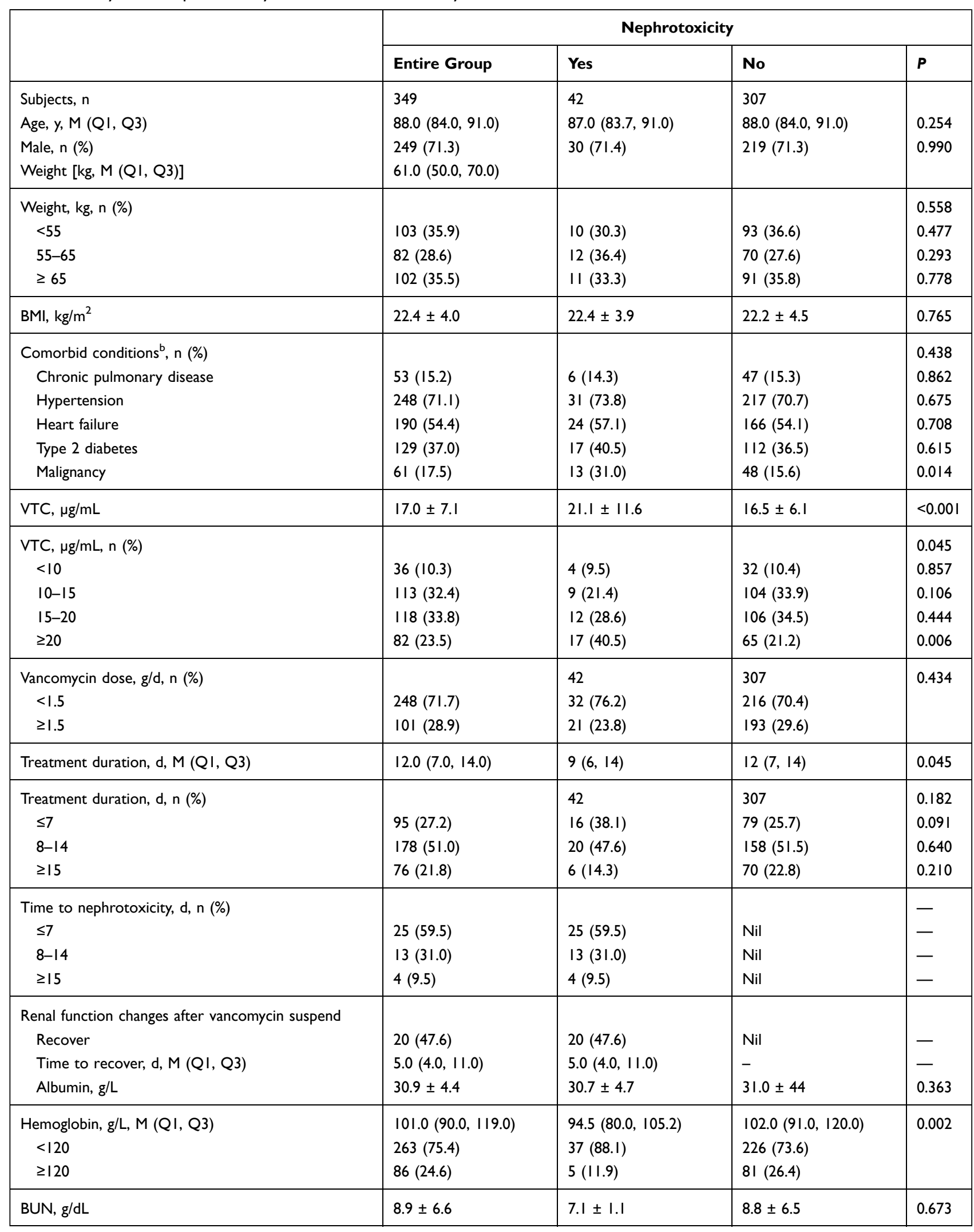

(Continued) 
Table 3 (Continued).

\begin{tabular}{|c|c|c|c|c|}
\hline & \multicolumn{4}{|c|}{ Nephrotoxicity } \\
\hline & Entire Group & Yes & No & $P$ \\
\hline SCr, g/dL, M (QI, Q3) & & & & \\
\hline Baseline $^{c}$ & $67.0(54.0,91.4)$ & $56.5(47.3,89.9)$ & $67.8(54.7,91.7)$ & 0.094 \\
\hline End of therapy ${ }^{d}$ & $68.3(52.7,93.3)$ & I $28.6(87.6, \mid$ I83.I $)$ & $65.0(51.0,86.4)$ & 0.002 \\
\hline $\mathrm{SCr}$ increase $\geq 0.5 \mathrm{mg} / \mathrm{dL}$ & $27(7.7)$ & $27(64.3)$ & Nil & $<0.001$ \\
\hline $\mathrm{SCr}$ increase from baseline $>50 \%$ & $37(10.6)$ & $37(88.1)$ & Nil & $<0.001$ \\
\hline eGFR, mL/min/l.73 $\mathrm{m}^{2}, \mathrm{n}(\%)$ & & & & 0.002 \\
\hline$\geq 90$ & $81(23.2)$ & $18(42.9)$ & $63(20.5)$ & 0.001 \\
\hline $60-89$ & $20 \mathrm{I}(57.6)$ & $15(35.7)$ & $186(60.6)$ & 0.002 \\
\hline $20-59$ & $67(19.2)$ & $9(2 \mid .4)$ & $58(18.9)$ & 0.696 \\
\hline Concomitant drugs ${ }^{\mathrm{e}}$, n (\%) & & & & 0.236 \\
\hline Aminoglycosides & $17(5.0)$ & $5(12.2)$ & $12(4.1)$ & 0.026 \\
\hline Diuretics & $77(22.1)$ & $9(21.4)$ & $68(22.1)$ & 0.916 \\
\hline NSAIDs & $4(1.1)$ & Nil & $4(\mathrm{I} .3)$ & 0.457 \\
\hline ACEI/ARB & $35(10.0)$ & $5(11.9)$ & $30(9.8)$ & 0.666 \\
\hline
\end{tabular}

Notes: a Shown as n (\%), unless indicated otherwise; ${ }^{b}$ One patient may have more than one comorbid disease; ${ }^{\mathrm{c} D a t a}$ collected at initiation of vancomycin treatment; ${ }^{\mathrm{d} D a t a}$ collected within 72 hours after vancomycin therapy completed; ${ }^{e}$ Four people used more than one drug to reduce renal perfusion simultaneously: 2 patients with aminoglycosides and diuretics, one patient with aminoglycosides and NSAIDs, and one patient with diuretics and NSAIDs.

Abbreviations: ACEI, angiotensin converting enzyme inhibitor; ARB, angiotensin receptor blocker; BUN, blood urea nitrogen; CKD, chronic kidney disease; eGFR, estimated glomerular filtration rate; NSAIDs, non-steroidal anti-inflammatory drugs; VTC, vancomycin trough concentration.

AUC/MIC index have been documented significantly higher in this particular population in some studies. Elevated vancomycin levels and AUC/MIC index increase the risk of nephrotoxicity. ${ }^{35}$ Vancomycin-associated nephrotoxicity is usually reversible, with a low incidence of residual damage if the vancomycin is withdrawn in time or doses are appropriately adjusted after renal damage occurs. According to a literature review by Elyasi et al, ${ }^{1} 44$ to $75 \%$ of patients with nephrotoxicity recovered within one week after vancomycin was discontinued. However, some studies indicated that not all critically ill patients could fully recover from vancomycin-

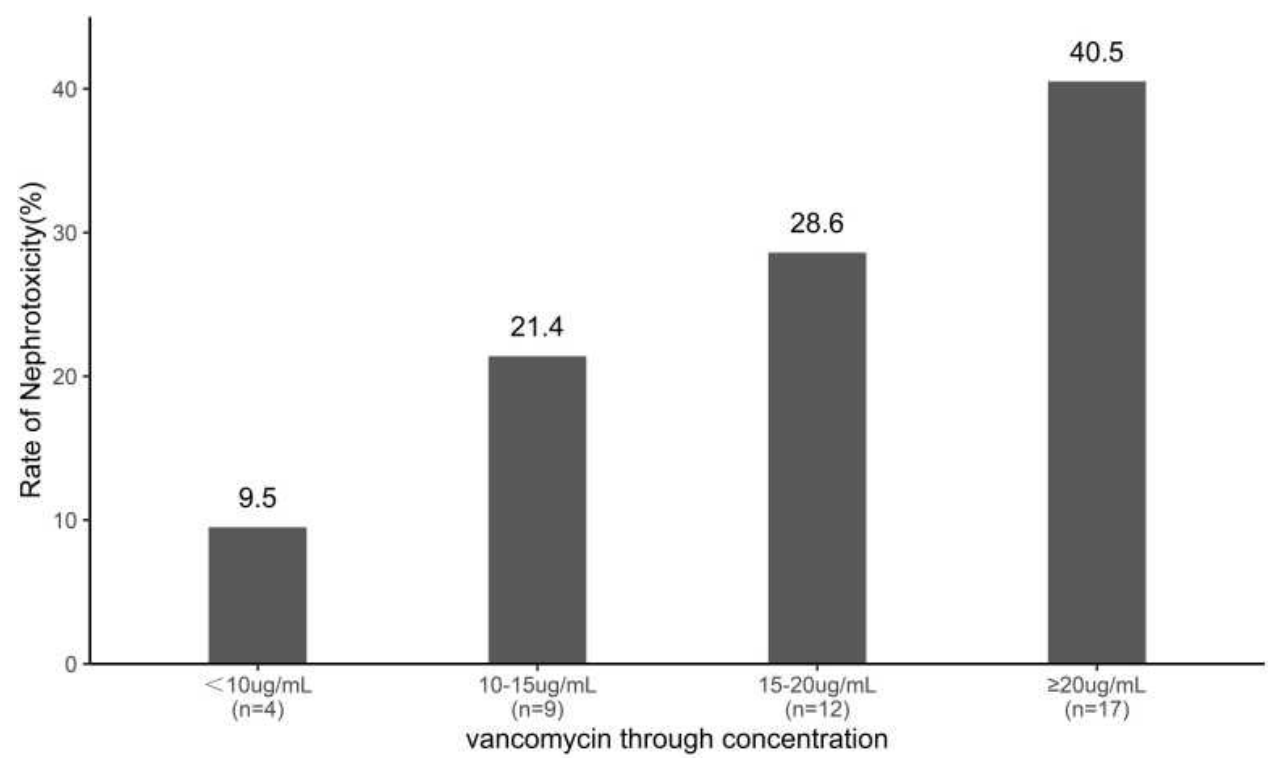

Figure 2 Rates of nephrotoxicity at VTCs $<10,10-15,15-20$, and $\geq 20 \mu g / m L$. 
Table 4 Multivariate Logistic Analysis for Nephrotoxicity in Elderly Patients on Vancomycin Therapy

\begin{tabular}{|c|c|c|c|c|}
\hline & $\boldsymbol{\beta}$ & SE & OR (95\% Cl) & $P$ value \\
\hline $\mathrm{VTC}^{\mathrm{a}}, \mu \mathrm{g} / \mathrm{mL}$ & 0.904 & 0.400 & $2.469(1.127-5.408)$ & 0.024 \\
\hline Aminoglycosides ${ }^{b}$ & 1.638 & 0.748 & $5.147(1.187-22.314)$ & 0.029 \\
\hline Malignancies $^{c}$ & 0.560 & 0.402 & $1.750(0.795-3.852)$ & 0.164 \\
\hline Hemoglobin ${ }^{d}$ & -0.674 & 0.515 & $0.510(0.186-1.398)$ & 0.190 \\
\hline \multicolumn{5}{|c|}{ eGFR, mL/min/l.73 m² } \\
\hline Reference, $\geq 90$ & - & - & - & - \\
\hline $60-90$ & -0.438 & 0.468 & $0.646(0.258-1.617)$ & 0.350 \\
\hline $20-60$ & 0.929 & 0.500 & $2.533(0.950-6.752)$ & 0.063 \\
\hline \multicolumn{5}{|c|}{ Treatment duration ${ }^{\mathrm{d}}$} \\
\hline Reference, $\leq 7$ & - & - & - & - \\
\hline $8-14$ & 0.458 & 0.517 & I.58I (0.574-4.350) & 0.376 \\
\hline$\geq 15$ & 1.176 & 0.558 & $3.24 \mid(1.086-9.672)$ & 0.035 \\
\hline
\end{tabular}

Notes: ${ }^{a} \mathrm{VTC}$ was categorized as $<15 \mu \mathrm{g} / \mathrm{mL}$ and $\geq 15 \mu \mathrm{g} / \mathrm{mL}$; ${ }^{\mathrm{b}}$ Aminoglycoside administration was categorized as with or without; ${ }^{\mathrm{C}}$ Malignancies consisted of solid and blood system tumors, categorized as with or without; ${ }^{d}$ Hemoglobin was continuous variable.

Abbreviations: $\beta$, regression coefficient; $\mathrm{Cl}$, confidence interval; OR, odds ratio; SE, standard error; VTC, vancomycin trough concentration.

associated nephrotoxicity, and even mild kidney injury could increase the hospital stay, healthcare costs, and mortality. ${ }^{33,36,37}$

In the present study, the time to nephrotoxicity was 7.5 \pm 4.5 days, and only $20(47.6 \%)$ of these elderly patients recovered after vancomycin was ended. The median time to recovery was 5 days (range, 4 to $11 \mathrm{~d}$ ). Some studies found no significant relation of nephrotoxicity to vancomycin treatment duration, ${ }^{11}$ but more often a positive result was found. Study by Hall et al showed that vancomycin duration greater than 15 days was an independent predictor of nephrotoxicity, with a 3.36-fold increased risk of nephrotoxicity relative to patients with shorter treatment duration. ${ }^{38}$ Another study conducted by Jeffres et al indicated that patients with longer than 14 days vancomycin treatment are more likely to developed renal toxicity (45.0\% vs $20.4 \%$; $P=0.011) .{ }^{39}$ Consistent with their finding, our study also showed that prolonged vancomycin treatment duration ( $\geq 15$ days) was independent association with increased nephrotoxicity. This suggests that it is not only the critically ill who may not recover renal function after vancomycin-associated nephrotoxicity, but also the very old. Vancomycin should be applied with caution and attentive monitoring.

In addition to VTC, the univariate analysis also showed that aminoglycosides administered concomitantly with vancomycin was associated with a 4.3 -fold greater risk of nephrotoxicity. Cano et $\mathrm{al}^{40}$ reported that this risk was 2.6-fold, after adjusting for potential confounders. Other studies also showed a positive association between concomitant aminoglycosides and vancomycin and nephrotoxicity in elderly patients. ${ }^{29,41,42}$ Besides aminoglycosides, other studies also indicated that concomitant use of renal hypoperfusion medications such as loop/thiazide diuretics, ${ }^{34}$ ACEI/ARBs, ${ }^{43}$ NSAIDs $^{44}$ and potential toxins include amphotericin B, trimethoprim-sulfamethoxazole, acyclovir and calcineurin inhibitors may increase the risk of nephrotoxicity, but these results were controversial. ${ }^{1,11}$ However, due to limited data in our study, not all the above agents were included in our analysis, and no statistical significance was found between some renal hypoperfusion medications or potential toxins and nephrotoxicity, except for aminoglycosides. The mechanism of nephrotoxicity when using vancomycin concomitant with aminoglycosides remains uncertain. Some preclinical studies and human data support the potential synergistic nephrotoxicity of vancomycin and aminoglycosides, ${ }^{45}$ but whether the enhanced rate of nephrotoxicity reported with vancomycin use in combination with aminoglycosides is the result of severity of underlying illness, the nephrotoxicity of aminoglycosides itself is not clearly. ${ }^{11}$ Therefore, in very old patients, multiple factors should be considered when making treatment decisions to improve vancomycin efficacy and reduce the risk of nephrotoxicity.

Three limitations should be noted. This was a singlecenter retrospective observational study, and prospective and multicenter studies are needed to confirm the findings. Secondly, this analysis did not include factors that may affect the prognosis of elderly patients with infection, such as APACHE II score and frailty. Finally, 
because of the small number of patients, a sub-group analysis of the effect of body mass index was not performed.

\section{Conclusion}

Regarding vancomycin treatment in the very old, this study found that nephrotoxicity, but not improved outcomes, were associated with elevated VTC, and VTC $\geq 20 \mu \mathrm{g} / \mathrm{mL}$ appears to suggest a worsened prognosis. Vancomycin treatment is likely to fail in the presence of increased BUN and heart failure. Risk factors of nephrotoxicity include higher VTC, prolonged treatment, and concomitant administration of aminoglycosides.

\section{Abbreviations}

ACEI, angiotensin converting enzyme inhibitor; APACHE II, Acute Physiology and Chronic Health Evaluation II; ARB, angiotensin receptor blocker; $\beta$, regression coefficient; BMI, body mass index; BUN, blood urea nitrogen; CI, confidence interval; CKD, chronic kidney disease; CNS, coagulase negative staphylococcus; eGFR, estimated glomerular filtration rate; OR, odds ratio; MRSA, methicillin-resistant Staphylococcus aureus; NSAID, nonsteroidal anti-inflammatory drug; SCr, serum creatinine; SE, standard error; VTC, vancomycin trough concentration.

\section{Data Sharing Statement}

The data used and/or analyzed during the current study are available from the corresponding author on reasonable request.

\section{Acknowledgments}

This work was supported by grants from Capital's Funds for Health Improvement and Research [Grant No: 20202-1101]. We thank Dr. Wei Wang for his support in data collection. We thank Dr. Tang for contributing to the development of this article, and Dr. Tian for Complete Medical Communications.

\section{Disclosure}

Dr Chunyan Jiang reports grants from Capital's Funds for Health Improvement and Research (2020-2-1101), during the conduct of the study. The authors report no other conflicts of interest in this work.

\section{References}

1. Elyasi S, Khalili H, Dashti-Khavidaki S, et al. Vancomycin-induced nephrotoxicity: mechanism, incidence, risk factors and special populations. A literature reviews. Eur $J$ Clin Pharmacol. 2012;68:1243-1255. doi:10.1007/s00228-012-1259-9

2. Rybak MJ, Le J, Lodise TP, et al. Therapeutic monitoring of vancomycin for serious methicillin-resistant Staphylococcus aureus infections: a revised consensus guideline and review by the American society of health-system pharmacists, the infectious diseases society of America, the pediatric infectious diseases society, and the society of infectious diseases pharmacists. Am $J$ Health Syst Pharm. 2020;77:835-864. doi:10.1093/ajhp/zxaa036

3. Bruniera FR, Ferreira FM, Saviolli LR, et al. The use of vancomycin with its therapeutic and adverse effects: a review. Eur Rev Med Pharmacol Sci. 2015;19:694-700.

4. Holmes NE, Tong SY, Davis JS, et al. Treatment of methicillin-resistant Staphylococcus aureus: vancomycin and beyond. Semin Respir Crit Care Med. 2015;36:17-30. doi:10.1055/ s-0034-1397040

5. Rybak MJ, Lomaestro BM, Rotschafer JC, et al. Vancomycin therapeutic guidelines: a summary of consensus recommendations from the infectious diseases society of America, the American society of health-system pharmacists, and the society of infectious diseases pharmacists. Clin Infect Dis. 2009;49:325-327. doi:10.1086/600877

6. Wei WX, Qin XL, Cheng DH, et al. Retrospective analysis of vancomycin treatment outcomes in Chinese paediatric patients with suspected Gram-positive infection. $J$ Clin Pharm Ther. 2016;41:650-656. doi:10.1111/jcpt.12437

7. Shime N, Saito N, Bokui M, et al. Clinical outcomes after initial treatment of methicillin-resistant Staphylococcus aureus infections. Infect Drug Resist. 2018;11:1073-1081. doi:10.2147/IDR.S159447

8. Álvarez R, López Cortés LE, Molina J, et al. Evaluation of vancomycin serum trough concentrations and outcomes in meticillin-resistant Staphylococcus aureus bacteraemia. Int $J$ Antimicrob Agents. 2012;40:474-475. doi:10.1016/j. ijantimicag.2012.06.020

9. Jeffres MN. The whole price of vancomycin: toxicities, troughs, and time. Drugs. 2017;77:1143-1154. doi:10.1007/s40265-017-0764-7

10. van Hal SJ, Paterson DL, Lodise TP. Systematic review and meta-analysis of vancomycin-induced nephrotoxicity associated with dosing schedules that maintain troughs between 15 and 20 milligrams per liter. Antimicrob Agents Chemother. 2013;57:734-744. doi:10.1128/AAC.01568-12

11. Filippone EJ, Kraft WK, Farber JL. The nephrotoxicity of vancomycin. Clin Pharmacol Ther. 2017;102(3):459-469. doi:10.1002/cpt.726

12. Sakamoto Y, Yano T, Hanada Y, et al. Vancomycin induces reactive oxygen species dependent apoptosis via mitochondrial cardiolipin peroxidation in renal tubular epithelial cells. Eur J Pharmacol. 2017;800:48-56. doi:10.1016/j.ejphar.2017.02.025

13. King DW, Smith MA. Proliferative responses observed following vancomycin treatment in renal proximal tubule epithelial cells. Toxicology. 2004;18:797-803.

14. Ray S, Haikal A, Hammoud KA, et al. Vancomycin and the risk of AKI: a systematic review and meta-analysis. Clin J Am Soc Nephrol. 2016;11:2132-2140. doi:10.2215/CJN.05920616

15. Corsonello A, Pedone C, Incalzi RA. Age-related pharmacokinetic and pharmacodynamic changes and related risk of adverse drug reactions. Curr Med Chem. 2010;17:571-584. doi:10.2174/ 092986710790416326

16. Mizokami F, Shibasaki M, Yoshizue Y, et al. Pharmacodynamics of vancomycin in elderly patients aged 75 years or older with methicillin-resistant Staphylococcus aureus hospital-acquired pneumonia. Clin Interv Aging. 2013;8:1015-1021. doi:10.2147/CIA. S50238 
17. Sánchez JL, Dominguez AR, Lane JR, et al. Population pharmacokinetics of vancomycin in adult and geriatric patients: comparison of eleven approaches. Int J Clin Pharmacol Ther. 2010;48:525-533. doi: $10.5414 / \mathrm{CPP} 48525$

18. Bourguignon L, Cazaubon Y, Debeurme G, et al. Pharmacokinetics of vancomycin in elderly patients aged over 80 years. Antimicrob Agents Chemother. 2016;60:4563-4567. doi:10.1128/AAC.00303-16

19. Walger P, Heppner HJ. Calculated parenteral initial therapy of bacterial infections: antibiotic treatment in the elderly. GMS Infect Dis. 2020;8:Doc05.

20. Prybylski JP. Vancomycin trough concentration as a predictor of clinical outcomes in patients with Staphylococcus aureus bacteremia: a meta-analysis of observational studies. Pharmacotherapy. 2015;35:889-898. doi:10.1002/phar.1638

21. Huang $\mathrm{M}$, $\mathrm{Wu} \mathrm{H}$, Zhou $\mathrm{J}$, et al. Efficacy of vancomycin on gram-positive bacterial infection in elderly critical patients and risk factors associated with nephrotoxicity. Arch Iran Med. 2018;21:349-355.

22. Hermsen ED, Hanson M, Sankaranarayanan J, et al. Clinical outcomes and nephrotoxicity associated with vancomycin trough concentrations during treatment of deep-seated infections. Expert Opin Drug Saf. 2010;9:9-14. doi:10.1517/14740330903413514

23. Steinmetz T, Eliakim-Raz N, Goldberg E, et al. Association of vancomycin serum concentrations with efficacy in patients with MRSA infections: a systematic review and meta-analysis. Clin Microbiol Infect. 2015;21:665-673. doi:10.1016/j.cmi.2015.04.003

24. Tongsai S, Koomanachai P. The safety and efficacy of high versus low vancomycin trough levels in the treatment of patients with infections caused by methicillin-resistant Staphylococcus aureus: a meta-analysis. BMC Res Notes. 2016;9:455. doi:10.1186/s13104-016-2252-7

25. Kullar R, Davis SL, Levine DP, et al. Impact of vancomycin exposure on outcomes in patients with methicillin-resistant Staphylococcus aureus bacteremia: support for consensus guidelines suggested targets. Clin Infect Dis. 2011;52:975-981. doi:10.1093/cid/cir124

26. Cheong JY, Makmor-Bakry M, Lau CL, et al. The relationship between trough concentration of vancomycin and effect on methicillin-resistant Staphylococcus aureus in critically ill patients. S Afr Med J. 2012;102:616-619. doi:10.7196/SAMJ.5343

27. Moore $\mathrm{CL}, \mathrm{Lu} \mathrm{M}$, Cheema F, et al. Prediction of failure in vancomycin-treated methicillinresistant Staphylococcus aureus bloodstream infection: a clinically useful risk stratification tool. Antimicrob Agents Chemother. 2011;55:4581-4588. doi:10.1128/ AAC.00115-11

28. Walraven CJ, North MS, Marr-Lyon L, et al. Site of infection rather than vancomycin MIC predicts vancomycin treatment failure in methicillin-resistant Staphylococcus aureus bacteraemia. J Antimicrob Chemother. 2011;66:2386-2392. doi:10.1093/jac/dkr301

29. Park SJ, Lim NR, Park HJ, et al. Evaluation of risk factors for vancomycin-induced nephrotoxicity. Int $J$ Clin Pharm. 2018;40:1328-1334. doi:10.1007/s11096-018-0634-8

30. Lodise TP, Patel N, Lomaestro BM, et al. Relationship between initial vancomycin concentration-time profile and nephrotoxicity among hospitalized patients. Clin Infect Dis. 2009;49:507-514. doi: $10.1086 / 600884$

31. Chuma M, Makishima M, Imai T, et al. Relationship between initial vancomycin trough levels and early-onset vancomycin-associated nephrotoxicity in critically ill patients. Ther Drug Monit. 2018;40:109-114. doi:10.1097/FTD.0000000000000459
32. Pan C, Wen A, Li X, et al. Development and validation of a risk prediction model of vancomycin-associated nephrotoxicity in elderly patients: a pilot study. Clin Transl Sci. 2020;13:491-497. doi: $10.1111 /$ cts. 12731

33. Singbartl K, Kellum JA. AKI in the ICU: definition, epidemiology, risk stratification, and outcomes. Kidney Int. 2012;81:819-825. doi:10.1038/ki.2011.339

34. Hirai T, Hanada K, Kanno A, et al. Risk factors for vancomycin nephrotoxicity and time course of renal function during vancomycin treatment. Eur J Clin Pharmacol. 2019;75:859-866. doi:10.1007/ s00228-019-02648-7

35. Yahav D, Abbas M, Nassar L, et al. Attention to age: similar dosing regimens lead to different vancomycin levels among older and younger patients. Age Ageing. 2019;49(1):26-31. doi:10.1093/ageing/afz135

36. Neely MN, Kato L, Youn G, et al. Prospective trial on the use of trough concentration versus area under the curve to determine therapeutic vancomycin dosing. Antimicrob Agents Chemother. 2018;62(2).

37. Linder A, Fjell C, Levin A, et al. Small acute increases in serum creatinine are associated with decreased long-term survival in the critically ill. Am J Respir Crit Care Med. 2014;189:1075-1081. doi:10.1164/rccm.201311-2097OC

38. Hall RN, Hazlewood KA, Brouse SD, et al. Empiric guideline-recommended weight-based vancomycin dosing and nephrotoxicity rates in patients with methicillin-resistant Staphylococcus aureus bacteremia: a retrospective cohort study. BMC Pharmacol Toxicol. 2013;14:12. doi:10.1186/2050-6511-14-12

39. Jeffres MN, Isakow W, Doherty JA, et al. A retrospective analysis of possible renal toxicity associated with vancomycin in patients with health care-associated methicillin-resistant Staphylococcus aureus pneumonia. Clin Ther. 2007;29(6):1107-1115. doi:10.1016/j. clinthera.2007.06.014

40. Cano EL, Haque NZ, Welch VL, et al. Incidence of nephrotoxicity and association with vancomycin use in intensive care unit patients with pneumonia: retrospective analysis of the IMPACT-HAP Database. Clin Ther. 2012;34:149-157. doi:10.1016/j. clinthera.2011.12.013

41. Rybak MJ, Albrecht LM, Boike SC, et al. Nephrotoxicity of vancomycin, alone and with an aminoglycoside. J Antimicrob Chemother. 1990;25:679-687. doi:10.1093/jac/25.4.679

42. Perazella MA. Drug-induced acute kidney injury: diverse mechanisms of tubular injury. Curr Opin Crit Care. 2019;25:550-557. doi:10.1097/MCC.0000000000000653

43. Matson KL, Shaffer CL, Beck GL, et al. Assessment of initial serum vancomycin trough concentrations and their association with initial empirical weight-based vancomycin dosing and development of nephrotoxicity in children: a multicenter retrospective study. Pharmacotherapy. 2015;35(3):337-343. doi:10.1002/phar.1552

44. Gambaro G, Perazella MA. Adverse renal effects of anti-inflammatory agents: evaluation of selective and nonselective cyclooxygenase inhibitors. J Intern Med. 2003;253(6):643-652. doi:10.1046/j.1365-2796.2003.01146.x

45. Wood CA, Kohlhepp SJ, Kohnen PW, et al. Vancomycin enhancement of experimental tobramycin nephrotoxicity. Antimicrob Agents Chemother. 1986;30(1):20-24. doi:10.1128/AAC.30.1.20 


\section{Publish your work in this journal}

Clinical Interventions in Aging is an international, peer-reviewed journal focusing on evidence-based reports on the value or lack thereof of treatments intended to prevent or delay the onset of maladaptive correlates of aging in human beings. This journal is indexed on PubMed Central, MedLine, CAS, Scopus and the Elsevier
Bibliographic databases. The manuscript management system is completely online and includes a very quick and fair peer-review system, which is all easy to use. Visit http://www.dovepress.com/ testimonials.php to read real quotes from published authors.

Submit your manuscript here: https://www.dovepress.com/clinical-interventions-in-aging-journal 